\title{
Inelasticity for hadron-carbon nucleus collisions from emulsion chamber
}

\author{
G. Wilk ${ }^{\mathrm{a}}$ and Z.Włodarczyk $\mathrm{k}^{\mathrm{b} *}$ \\ aThe Andrzej Soltan Institute for Nuclear Studies, Nuclear Theory Department, Warsaw, Poland \\ email: wilk@fuw.edu.pl \\ ${ }^{\mathrm{b}}$ Institute of Physics, Pedagogical University, Kielce, Poland \\ email: wlod@pu.kielce.pl
}

The inelasticity of hadron-carbon collisions for energies exceeding $100 \mathrm{TeV}$ is estimated from the carbonemulsion chamber data at Pamirs to be $\left\langle K_{C}\right\rangle=0.65 \pm 0.08$. When combined with data on hadron-lead collisions taken at the same energy range it results in the $K \sim A^{0.086}$ mass number dependence of inelasticity. The evaluated partial inelasticity for secondary $(\nu>1)$ interactions, $K_{\nu>1} \simeq 0.2$, suggests that most of the energy is lost in the first interaction.

\section{INTRODUCTION}

The inelasticity of hadronic reactions, understood as the fraction of the incident beam energy spent on the production of secondaries, is (next to the inelastic cross section) the most significant variable for all cosmic ray experiments involved in cascade developments [1.22]. Unfortunately, in the energy region exceeding a few hundreds of $\mathrm{GeV}$ there are no accelerator data on inelasticity on nuclear targets and only rough indications from cosmic ray experiments are available [1,2]. Recently [3] the inelasticity in hadron-lead collisions was estimated in the energy region exceeding 100 $\mathrm{TeV}$. In this contribution we present similar analysis performed for $h-C$ collisions observed in carbon emulsion chamber (EC) exposed to cosmic rays at the Pamirs.

This EC consists of $\Gamma$-block of $6 \mathrm{~cm} P b(0.35 \lambda$, 10.5 c.u.) and two $H$-blocks of carbon layer of 60 $\mathrm{cm}$ thickness $\left(66 \mathrm{~g} / \mathrm{cm}^{2}, 0.9 \lambda, 2.5\right.$ c.u.) each followed by $5 \mathrm{~cm}$ of lead-emulsion sandwiches. In EC (which is a shallow calorimeter) only the energy transfered to the electromagnetic component is measured, i.e., $E_{h}^{\gamma}=K_{\gamma} \cdot E_{h}$, and in the hadronic block a given nuclear-electromagnetic

\footnotetext{
*Talk given at the Xth International Symposium on Very High Energy Cosmic Ray Interactions, Laboratori Nazionali del Gran Sasso, 12-17 July 1998, Assergi, Italy, to be published in the proceedings (Nucl. Phys. B (Proc. Suppl.)).
}

cascade (NEC) produces spots with optical den-

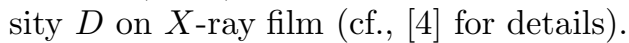

Such structure of the carbon EC allows for a relatively straightforward estimation of the total inelasticity for $h-C$ interactions [5]. The proposed method is uses the repeated registration of the same cascade in the two subsequent hadronic blocks. If $N_{1}$ denote the number of cascades registered in the first hadronic block with visible energy $E_{1}>\left(E_{h}^{\gamma}\right)_{1}$ and $N_{2}$ the number of cascades repeated registered in second hadronic block with $E_{2}$ above the threshold $\left(E_{h}^{\gamma}\right)_{2}$, then the ratios

$\eta=\frac{N_{2}}{N_{1}} \quad$ and $\quad \epsilon=\frac{E_{2}}{E_{1}}$

are sensitive to total inelasticity $K$. The weak dependence of these quantities on the methodical errors and ease with which the experimental data may by obtained render this method very useful and promising for possible future applications.

\section{INELASTICITY FOR CARBON TAR- GET}

The experimental data collected from $110 \mathrm{~m}^{2}$ carbon EC contain $N_{1}=70$ hadrons with energies $E_{1}>30 \mathrm{TeV}$ and $N_{2}=24$ hadrons with energies $\left.E_{2}>2 \mathrm{TeV}\right)$. They give the value of $\eta=0.27 \pm 0.06$ (at energy threshold $E_{2}>4 \mathrm{TeV}$, being free from the detection bias) and the energy ratio $\epsilon=0.24 \pm 0.07$. These data have been 
then recalculated by using the simulated $D\left(E_{h}^{\gamma}\right)$

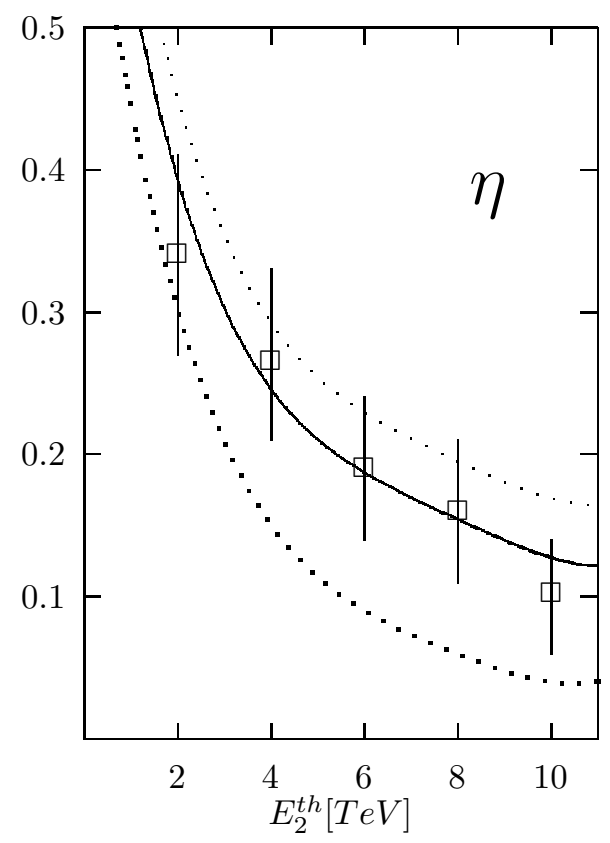

Figure 1. Dependence of $\eta=N_{2} / N_{1}$ on the energy threshold $E t h_{2}$ in the second hadronic block for: $\langle K\rangle=0.65$ (solid line), $\langle K\rangle=0.50$ (dotted line) and for $\langle K\rangle=0.80$ (black dots), compared with the experimental data.

dependence [4]. The repeated registrations of hadron has been simulated by the Monte-Carlo event generator. Primary hadrons (assumed to consist of $75 \%$ nucleons and $25 \%$ pions) were sampled from the power spectrum representing distribution of the initial energy with a differential slope equal to $\gamma=3$. The gamma quanta and electrons above $0.01 \mathrm{TeV}$, reaching the detection level within the radius of $5 \mathrm{~mm}$, were recorded and the corresponding optical densities were calculated within the radii utilized in the experiment. Only cascades with the energies above $E_{1}=30 \mathrm{TeV}$ and $E_{2}=2 \mathrm{TeV}$ were selected.
The ratio $\eta$ of the number of hadrons repeatedly registered in two hadronic blocks and the number of all hadrons registered in the first hadronic block is presented in Fig.1 for different total inelasticities: $\langle K\rangle=0.5,0.65$ and 0.80 . Note that the ratio $\eta$ is more sensitive to the mean value of inelasticity $\langle K\rangle$ than the energy ratio $\epsilon$, shown in Fig.2. The comparison of experimental data with simulated dependences indicates that $\left\langle K_{C}\right\rangle=0.65 \pm 0.08$ for hadron-carbon nucleus collisions at the hadron energies of above $\sim 100$ $\mathrm{TeV}$ is the most probably choice for the mean value of inelasticity for hadron-carbon collisions.

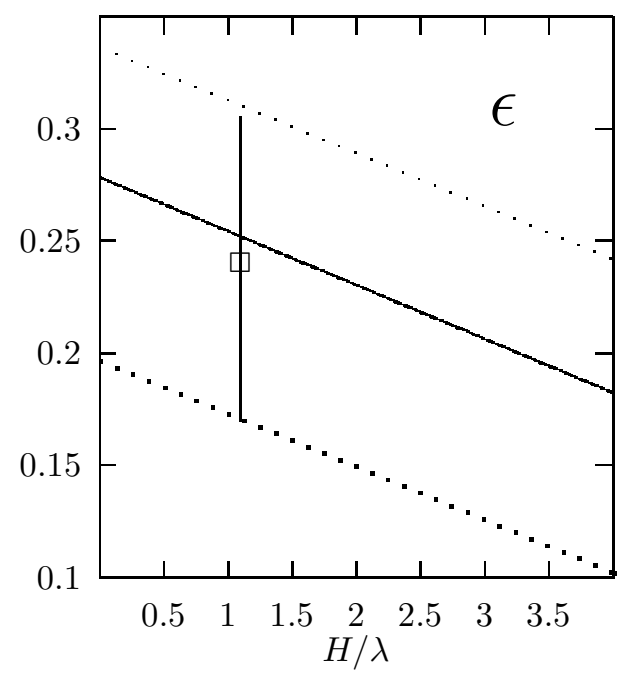

Figure 2. Dependence of $\epsilon=E_{2} / E_{1}$ on the thickness $H / \lambda$ of carbon target (the plotted curves correspond to different $\langle K\rangle$ as in Fig. 1).

This result can be now compared with $\left\langle K_{P b}\right\rangle=$ $0.83 \pm 0.17$ obtained in similar analysis of succesive hadron interactions registered in the thicklead-emulsion chamber at Pamirs [3]. Both results lead to the $K \simeq A^{0.086}$ mass number dependence of inelasticity. If we extrapolate this $A$ dependence to $A=1$ we obtain hadron-proton 
inelasticity in this energy range being equal to $K_{h-p}=0.53$ (notice that the same value can be obtained from Eq. (3) below where $K_{1}=K_{h-p}$ ). Assuming further that $N_{\pi} / N_{h} \sim 0.25$ and that $K_{\pi-p}=1.5 K_{p-p}$, we obtain that in the energy range exceeding $100 \mathrm{TeV}$ inelasticity in protonproton collisions is equal to $K_{p-p}=0.46$ (which agrees with our earlier predictions [1,6]).

\section{PARTIAL INELASTICITY $K_{\nu}$}

Following Ref. [2] we shall now estimate the so called partial inelasticity $K_{\nu}$. In the framework of Glauber multiple scattering formalism [7]) it is defined by

$$
\langle 1-K\rangle=\sum_{\nu=1} P_{\nu}\left\langle 1-K_{\nu}\right\rangle^{\nu}
$$

where $P_{\nu}$ is the probability for encountering exactly $\nu$ wounded nucleons in a target of mass $A$ and $\left\langle 1-K_{\nu}\right\rangle$ is the mean elasticity of the leading hadron in collisions with exactly $\nu$ wounded nucleons. Assuming that $K_{\nu>1}=K_{2}$ the total elasticity can be written as

$$
\langle 1-K\rangle=\left(1-K_{1}\right) \sum_{\nu=1}\left\langle 1-K_{2}\right\rangle^{\nu-1} P_{\nu} .
$$

The ratio $\kappa$ of elasticities (given by eq.(3)) in collisions on $\mathrm{Pb}$ and $\mathrm{C}$ targets depends only on $K_{2}$ once the $P_{\nu}$ is known. Assuming Poisson distribution for the number of wounded nucleons, $\nu$, the value of $K_{2}$ for the expected mean number of wounded nucleons $\langle\nu\rangle=\frac{A \sigma_{h-p}}{\sigma_{h A}} \sim A^{1 / 3}$ and for the experimatally evaluated value of $\kappa=0.5$ is equal to $K_{2} \simeq 0.2$. (Notice tacid assumption made here that the ultimate identity of the final state nucleon is determined only once during the interaction with the nucleus - in [2] it means that $\beta=1$ for the parameter specifing the fraction of isospin preserving reactions).

\section{CONCLUDING REMARKS}

For hadron-carbon nucleus collisions in energy region exceeding $100 \mathrm{TeV}$ the inelasticity is estimated to be equal to $\left\langle K_{C}\right\rangle=0.65 \pm 0.08$. Our estimation of $K_{2}=0.2$ at energies above $100 \mathrm{TeV}$ is consistent with low energy data (cf.
Ref. [2]). Note that inequality $K_{\nu>1}<K_{1}$ is characteristic to all string-type interaction models (cf. Quark-Gluon String model [8] or Dual Parton Model [9]) whereas the SIBYLL model [2.10] predict much smaller value of $K_{2}$ in the examined energy region. It is important to notice that the new results for inelasticity on lead target, $\left\langle K_{h-P b}\right\rangle \simeq 0.6 \pm 0.05$ reported at this conference [11] when compared with our $\left\langle K_{h-C}\right\rangle$ result in $K_{2} \simeq 0$ and $\left\langle K_{h-p}\right\rangle=\left\langle K_{h-C}\right\rangle$ which exceeds noticeably the value $\left\langle K_{p p}\right\rangle \simeq 0.41$ as evaluated from the collider data [6].

\section{REFERENCES}

1. Yu.M.Shabelski, R.M.Weiner, G.Wilk and Z.Włodarczyk, J. Phys. G18 (1992) 1281 and references therein.

2. G.M.Frichter, T.K.Gaisser and T.Stanev, Phys. Rev. D56 (1997) 3135.

3. S.L.C.Barroso et al., Nucl. Phys. B Proc. Suppl. 52B (1997) 201.

4. A.Tomaszewski and Z.Włodarczyk, Proc. 19th Int. Cosmic Ray Conf., 6 La Jolla (1985) 431.

5. J.Nowicka, Z.Włodarczyk and A.Podgorzak, Proc. Int. Symp. on Cosmic Rays and Part. Phys., eds. A.Ohsawa, T.Yuda, Tokyo (1984)451.

6. A.Ohsawa, Prog. Theor. Phys. 92 (1994) 1005.

7. R.J.Glauber and G.Matthie, Nucl. Phys. B21 (1970) 135.

8. A.B.Kaidalov, K.A.TerMartirosyan and Yu.M.Shabelsky, Yad. Fiz. 43 (1986) 1282 [Sov. J. Nucl. Phys. 43 (1986) $822]$.

9. A.Capella, U.Sukhatme, C.-I.Tan and J.Tran Thanh Van, Phys. Rep. 236 (1994) 225.

10. R.S.Fletcher, T.K.Gaisser, P.Lipari and T.Stanev, Phys. Rev. D50 (1994) 5710.

11. Cf., contribution by A.Ohsawa to this conference. 